\title{
LA-UR-16-26530
}

Approved for public release; distribution is unlimited.

Title: Introduction to Numerical Methods

Author(s): $\quad$ Schoonover, Joseph A

Intended for: Lecture for Parallel Computing Summer Research Internship at the National Security Education Center Online public release of talk slides.

Issued: 
Disclaimer:

Los Alamos National Laboratory, an affirmative action/equal opportunity employer, is operated by the Los Alamos National Security, LLC for the National Nuclear Security Administration of the U.S. Department of Energy under contract DE-AC52-06NA25396. By approving this article, the publisher recognizes that the U.S. Government retains nonexclusive, royalty-free license to publish or reproduce the published form of this contribution, or to allow others to do so, for U.S. Government purposes. Los Alamos National Laboratory requests that the publisher identify this article as work performed under the auspices of the U.S. Department of Energy. Los Alamos National Laboratory strongly supports academic freedom and a researcher's right to publish; as an institution, however, the Laboratory does not endorse the viewpoint of a publication or guarantee its technical correctness. 


\section{Numerical Methods}

Repetitive algorithms to obtain approximate solutions to mathematical problems

Sorting

Searching

Root Finding

Optimization

Interpolation

Extrapolation

Least squares regression

Eigenvalue Problems

Ordinary Differential Equations

Partial Differential Equations 


\section{Starting Simple}

\section{$c_{t}=\lambda c$}

Goal : Given an initial condition, use basic arithmetic to estimate the solution at a later time.

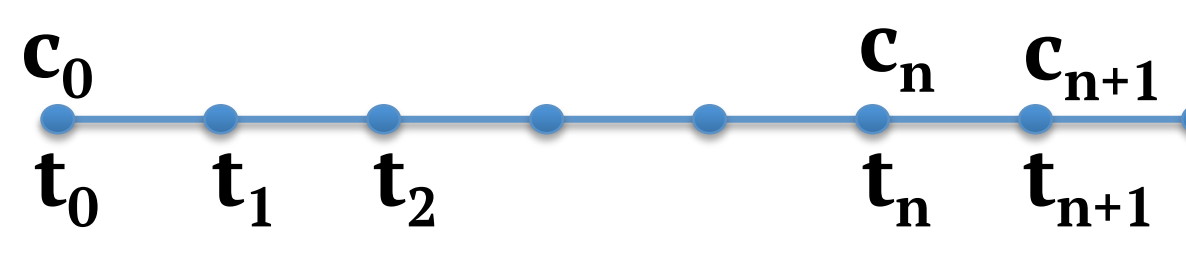




\section{Continuous to Discrete}

\section{$c_{t}=\lambda c$}

Use Taylor Series

$$
\left.c_{t}\right|_{t^{n}}=\frac{c^{n+1}-c^{n}}{\Delta t}+\left.\frac{\Delta t}{2} c_{t t}\right|_{\xi}, \quad \xi \in\left[t_{n}, t^{n+1}\right]
$$

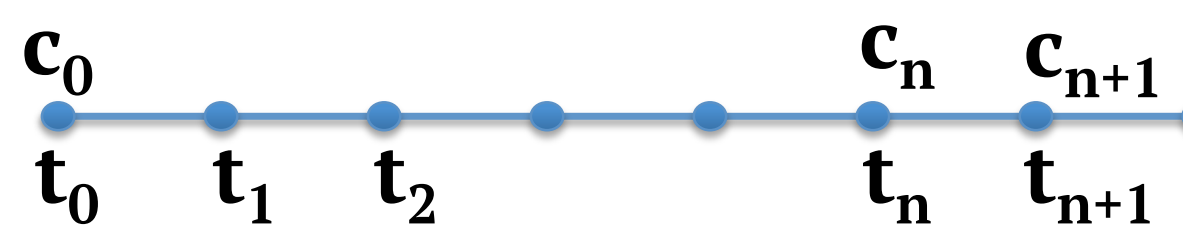




\section{Introducing Errors}

$$
c^{n+1}=c^{n}+\lambda \Delta t c^{n}-\underbrace{\left.\frac{\Delta t^{2}}{2} c_{t t}\right|_{\xi}}_{\text {TruncationError }}
$$

Approximate the solution by dropping the truncation error

$$
\begin{gathered}
\stackrel{\text { "Forward Euler" }}{\tilde{c}^{n+1}=\tilde{c}^{n}+\lambda \Delta t \tilde{c}^{n}}
\end{gathered}
$$

\section{How big can the time step be?}




\section{Time Step and Stability}

\section{How can we control the error growth?}

$$
\begin{aligned}
& \text { Error } \\
& E^{n}=c^{n}-\tilde{c}^{n} \\
& E^{n+1}=\underbrace{(1+\lambda \Delta t)}_{\text {Amplification }} E^{n}-\left(\left.\frac{\Delta t^{2}}{2} c_{t t}\right|^{\text {error acts as }}\right. \text { a source }
\end{aligned}
$$

Error can be amplified! 


\section{Time Step and Stability}

\section{How can we control the error growth?}

\section{Don't amplify the error!}

$$
|G|=\left|\frac{\tilde{c}^{n+1}}{\tilde{c}^{n}}\right|=\underbrace{|1+\lambda \Delta t|}_{\text {ForwardEuler }}<1
$$

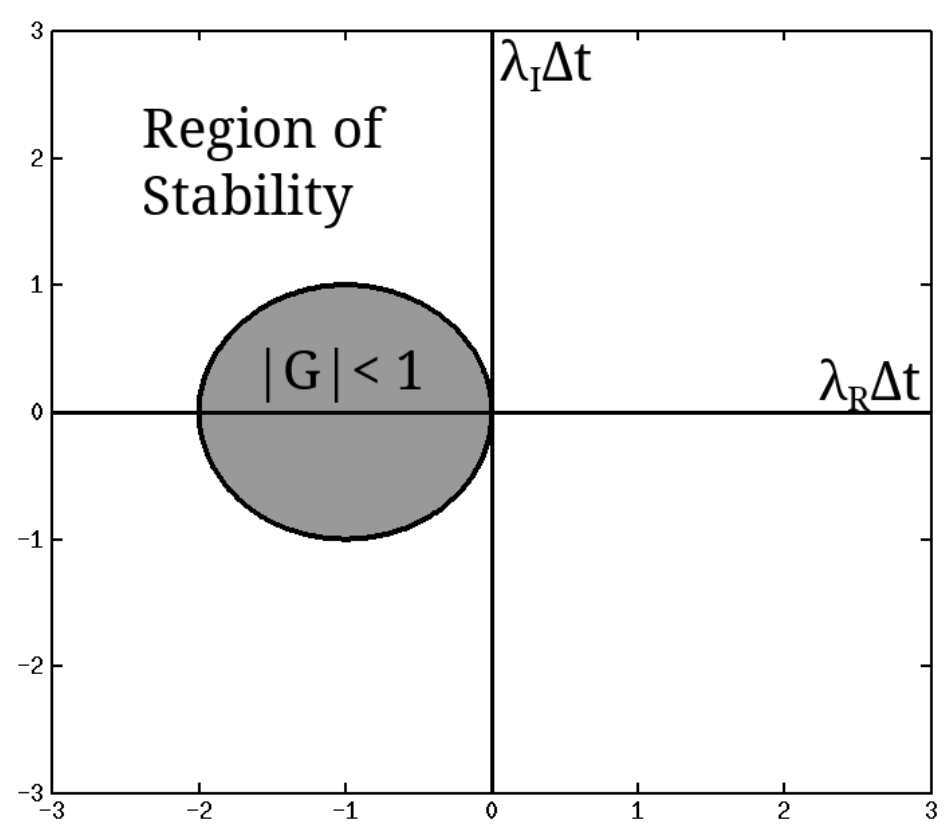




\section{Stability Diagrams and Implicit Integration Schemes}

$|G|=\underbrace{\left|\frac{1}{1-\lambda \Delta t}\right|}<1$ BackwardEuler

*Implicit schemes allow for larger time steps

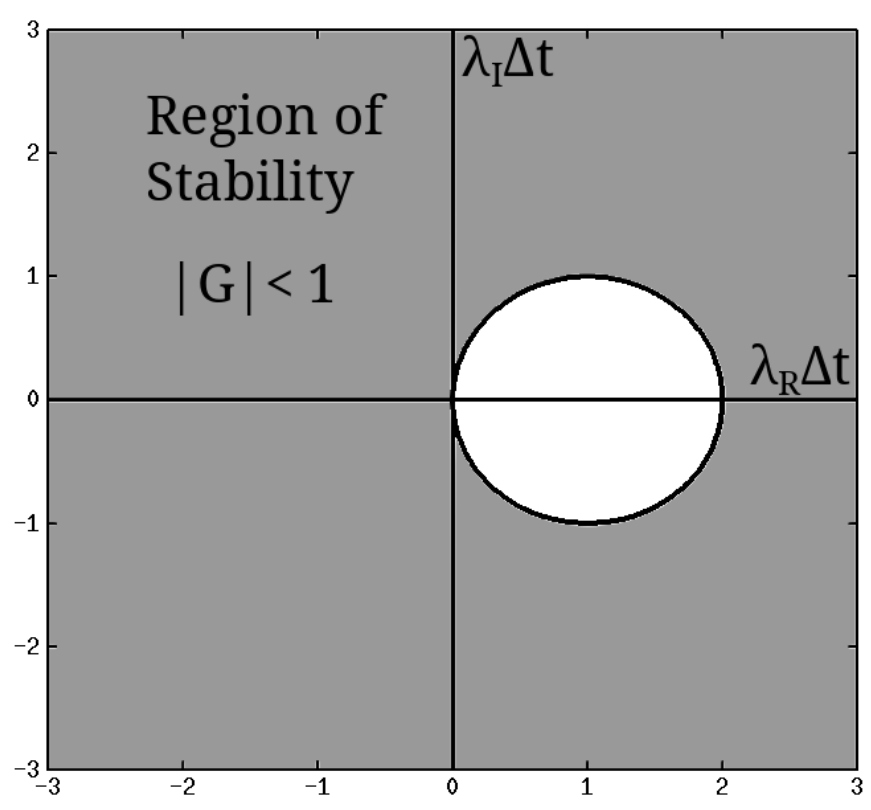

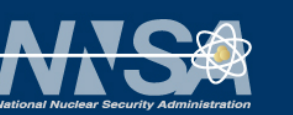
UNCLASSIFIED | 8 


\section{Convergence}

If the truncation error is $\mathcal{O}\left(\Delta t^{n}\right)$ and the integration scheme is stable,

then the approximate solution will converge to the exact solution as $\Delta t \rightarrow 0$ at a rate proportional to $\Delta t^{n}$. 


\section{Systems of ODEs}

$$
\begin{array}{ccc}
\text { PDE } & \begin{array}{c}
\text { Spatial } \\
\text { Discretization }
\end{array} & \text { ODE System } \\
\vec{s}_{t}+\nabla \cdot \vec{f}=\vec{q} \stackrel{\vec{c}_{t}}{\longrightarrow} \boldsymbol{A} \vec{c}
\end{array}
$$

\section{Stability}

- Decoupling = Diagonalization

$\boldsymbol{A}=\boldsymbol{P} \boldsymbol{D} \boldsymbol{P}^{-1}$

- Maximum eigenvalue determines time step restrictions

$\left(w_{i}\right)_{t}=\lambda_{i} w_{i}$ 


\section{Finite Difference Method Upwind Advection}

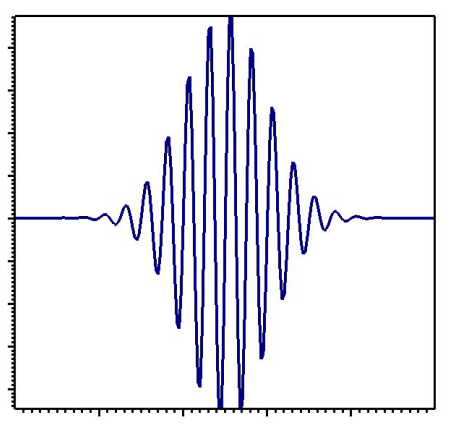

$$
c_{t}+u c_{x}=0
$$

Use Taylor Series for the spatial derivative

Restrict the truncation to zero at each node

$$
\left(\tilde{c}_{i}\right)_{t}=-\frac{u}{\Delta x}\left(\tilde{c}_{i}-\tilde{c}_{i-1}\right)
$$




\section{Finite Difference Method}

\section{Upwind Advection}

$$
\vec{c}_{t}=A \vec{c}
$$

Spatial discretization results in a sparse ODE system;

$$
\begin{gathered}
A_{i, i}=\frac{-u}{\Delta x}, A_{i, i-1}=\frac{u}{\Delta x} \\
\text { Integration by Forward Euler } \\
\tilde{c}_{i}^{n+1}=\tilde{c}_{i}^{n}-\frac{u \Delta t}{\Delta x}\left(\tilde{c}_{i}^{n}-\tilde{c}_{i-1}^{n}\right)
\end{gathered}
$$




\section{Finite Difference Method \\ Upwind Advection, Group Example}

$$
\tilde{c}_{i}^{n+1}=\tilde{c}_{i}^{n}-0.5\left(\tilde{c}_{i}^{n}-\tilde{c}_{i-1}^{n}\right)
$$

$i=N \quad i=1 \quad i=2 \quad i=3$

You each are assigned a process ID and three nodes

To advance the system

1. Exchange data with your "upstream neighbor"

2. Update the solution at your three nodes

3. Do your file I/O.

4. Wait for your neighbor to finish.

5. Repeat
Sample Solution Output

Solution.001.00000.data

$\begin{array}{lc}\text { i } & c \\ 1 & 0.000 \\ 2 & 0.000 \\ 3 & 0.000\end{array}$




\section{Finite Difference Method Implicit Upwind Advection}

$$
\tilde{c}_{i}^{n+1}=\tilde{c}_{i}^{n}-\frac{u \Delta t}{\Delta x} \underbrace{\left(\tilde{c}_{i}^{n+1}-\tilde{c}_{i-1}^{n+1}\right)}_{\text {Evaluated at the next time step }}
$$

Forward Integration requires matrix inversion

$$
(\boldsymbol{I}-\Delta t \boldsymbol{A}) \vec{c}^{n+1}=\vec{c}^{n}
$$




\section{General Discretization Overview}

- Approximate continuous functions by discrete observations

- Approximate derivative operators by difference operators

$$
\begin{aligned}
& \text { Spatial } \\
& \text { PDE } \\
& \text { Discretization }
\end{aligned}
$$

- Apply a suitable time integrator 


\section{Discretization Flavors}

Mesh-based

\begin{tabular}{|c|c|c|}
\hline Strong (Collocation) & Weak (Galerkin) & Conservative \\
\hline Finite Difference & Finite Element & Finite Volume \\
\hline Pseudo-Spectral & Spectral & \\
\hline & Spectral Element & \\
\hline
\end{tabular}

Mesh-free

Smoothed Particle Hydrodynamics

Element-free Galerkin

Discrete Vortex Method

Radial Basis Methods 


\section{Stability and Work Explicit Schemes}

- Wall-Time (Number of Floating Point Operations) /(FLOP/sec)

- Remember, stability requirements impose time-step restrictions

- We want to integrate to a known time, which requires $\mathrm{M}$ time-steps

$$
\lambda_{\max } \Delta t<C
$$

- The number of floating point operations per time step is proportional to the number of degrees of freedom

$$
M=\frac{T}{\Delta t}
$$

$$
W_{\text {float }} \propto \frac{N T \lambda(N)}{k_{F L O P S}}
$$




\section{Summary}

- I showed too many equations.

- I am sorry.

- Discretizations

- allow us to approximate solutions to mathematical models of physical systems using a repetitive algorithm.

- introduce errors that can lead to numerical instabilities if we are not careful.

- We barely scratched the surface of numerical methods.

- Let's talk more! 